\title{
Solutions of max-plus linear equations and large deviations
}

\author{
Marianne Akian, Stéphane Gaubert and Vassili Kolokoltsov
}

\begin{abstract}
We generalise the Gärtner-Ellis theorem of large deviations theory. Our results allow us to derive large deviation type results in stochastic optimal control from the convergence of generalised logarithmic moment generating functions. They rely on the characterisation of the uniqueness of the solutions of max-plus linear equations. We give an illustration for a simple investment model, in which logarithmic moment generating functions represent risk-sensitive values.
\end{abstract}

\section{INTRODUCTION}

Let $X, Y$ be two sets and $\overline{\mathbb{R}}=\mathbb{R} \cup\{ \pm \infty\}$ denote the extended real line. A Moreau conjugacy [1], associated to a kernel $b: X \times Y \rightarrow \overline{\mathbb{R}}$, is a map $B: \mathscr{F} \rightarrow \mathscr{G}$, where $\mathscr{F}$ and $\mathscr{G}$ are subsets of $\overline{\mathbb{R}}^{Y}$ and $\overline{\mathbb{R}}^{X}$ respectively, such that:

$$
B f(x)=\sup \{b(x, y)-f(y) \mid y \in Y\}, \quad \forall x \in X .
$$

Here $b(x, y)-f(y)$ is an abbreviation of $b(x, y)+(-f(y))$, with the convention that $-\infty$ is absorbing for addition. An example of Moreau conjugacy is the Legendre-Fenchel transform. Moreau conjugacies are instrumental in nonconvex duality, see [2, Chapter 11 , Section E],[3]. The set $\overline{\mathbb{R}}$ can be equipped with the semiring structure of $\overline{\mathbb{R}}_{\max }$, in which the addition is $(a, b) \mapsto \max (a, b)$ and the multiplication is $(a, b) \mapsto a+b$, with the same convention as above. If $B: \overline{\mathbb{R}}^{Y} \rightarrow \overline{\mathbb{R}}^{X}$ is a Moreau conjugacy, the map $f \mapsto$ $B(-f)$ is a max-plus linear operator with kernel. Max-plus linear operators with kernel arise in deterministic optimal control and asymptotics, and have been widely studied, see in particular [4], [5], [6], [7], [8], [9].

Given a map $g \in \mathscr{G}$ and a Moreau conjugacy $B: \mathscr{F} \rightarrow \mathscr{G}$, let us consider the problem:

$$
(\mathcal{P}): \text { Find } f \in \mathscr{F} \text { such that } B f=g,
$$

and more generally:

$$
\begin{array}{ll}
\left(\mathcal{P}^{\prime}\right): & \text { Find } f \in \mathscr{F} \text { such that } B f \leq g \\
& \text { and } B f(x)=g(x) \text { for all } x \in X^{\prime},
\end{array}
$$

where $X^{\prime} \subset X$ is given. In [10] we gave effective conditions on $g$ for the solution $f$ of $\left(\mathcal{P}^{\prime}\right)$ to exist and be unique, using generalised subdifferentials (associated to the Moreau conjugacy $B$ ). We characterised the existence and uniqueness of the solution of $\left(\mathcal{P}^{\prime}\right)$ in terms of coverings and minimal coverings by sets which are inverses of subdifferentials of $g$ (we recall these results in Section III). These conditions

Date: September 13, 2005. Prepared for CDC-ECC'05.

Marianne Akian and Stéphane Gaubert are with INRIA, Domaine de Voluceau, B.P. 105, 78153 Le Chesnay Cedex, France. Marianne.Akiandinria.fr, Stephane.Gauberteinria.fr

Vassili Kolokoltsov is with Institute of the Information Transmission Problems of the Russian Academy of Sciences, Moscow, Russia. vkolokefsmail.net extend, to the case of infinite sets $X$ and $Y$, the characterisations of the solutions of $(\mathcal{P})$ in terms of "minimal resolvent coverings" of $X$ which were first shown by Vorobyev [11, Theorem 2.6] and then developed by Zimmermann [12, Chapter 3], when $X$ and $Y$ are finite.

When $B$ is the Legendre-Fenchel transform, these results show that essentially smooth convex functions have a unique pre-image by the Legendre-Fenchel transform (see [10, Corollary 6.4]), a fact which is the essence of the classical Gärtner-Ellis theorem, see e.g. [13, Theorem 2.3.6,(c)] for a general presentation. Indeed, as we shall see in Section $\nabla$ Problem $\left(\mathcal{P}^{\prime}\right)$ arises naturally when looking for the rate function in large deviations.

Large deviation type asymptotics arise in optimal control when studying the long-term behaviour of some controlled stochastic process. For instance, assume that some real nonnegative controlled diffusion $X_{t}$, representing the wealth of an investor, has an asymptotic growth rate, which means that $\lim _{t \rightarrow+\infty} \frac{\log \left(X_{t}\right)}{t}$ exists almost surely, and assume that this growth rate satisfies a large deviation principle with factor $1 / t$, which means in loose terms that for "good" subsets $A$ of $\mathbb{R},-\frac{1}{t} \log P\left(\frac{\log \left(X_{t}\right)}{t} \in A\right)$ tends to $I(A):=\inf _{x \in A} I(x)$ for some rate function $I: \mathbb{R} \rightarrow[0,+\infty)$. Then, one may want to find a control minimising the rate $I(A)$. This problem was studied by Pham in [14], to which the reader is referred for more information.

In the present paper, we show how the results of [10] yield a characterisation of the rate function of a large deviation principle, generalising the Gärtner-Ellis theorem (Section $\nabla$. We also study rate functions in optimal stochastic control, such as the maximal long term growth rate of wealth described above. To develop these results in a unified way, we introduce (in Section III) the notion of quasi (maxplus) linear form. It includes as special cases, possibly up to a log-exp conjugacy: integrals with respect to finite measures, suprema of such integrals and idempotent integrals with respect to finite cost measures [8]. We then introduce (in Section IV] the notion of weak convergence of quasilinear forms, which generalises the large deviation principle of Varadhan. Finally, we illustrate the results on a simple investment model (Section VI).

\section{IMAGES AND PRE-IMAGES OF MOREAU CONJUGACIES}

We briefly recall some of the results of [10].

\section{A. Moreau conjugacies}

Let $X$ and $Y$ be two Hausdorff topological spaces. Denote by $\mathscr{F}$ the set of lower semicontinuous (1.s.c.) maps from $Y$ to $\overline{\mathbb{R}}$ and by $\mathscr{G}$ the set $\overline{\mathbb{R}}^{X}$ of all maps from $X$ to $\overline{\mathbb{R}}$. The sets 
$\mathscr{F}$ and $\mathscr{G}$ are endowed with the partial ordering of functions, for which they are complete lattices. Let $b: X \times Y \rightarrow \overline{\mathbb{R}}$ be a map which is lower semicontinuous in the second variable. Then the maps $B: \mathscr{F} \rightarrow \mathscr{G}$ and $B^{\circ}: \mathscr{G} \rightarrow \mathscr{F}$ defined by

$$
\begin{aligned}
B f(x) & =\sup \{b(x, y)-f(y) \mid y \in Y\} \forall f \in \mathscr{F}, x \in X, \\
B^{\circ} g(y) & =\sup \{b(x, y)-g(x) \mid x \in X\} \forall g \in \mathscr{G}, y \in Y
\end{aligned}
$$

are Moreau conjugacies [1] and they are conjugate to each other, or in duality, meaning that $\left(B, B^{\circ}\right)$ is a dual Galois connection (see [10, Theorem 2.1 and Example 2.7]). Moreover, by [10, Theorem 2.1], the map $b$ is uniquely determined by the Moreau conjugacy $B$. We call it the kernel of $B$. The kernel of $B^{\circ}$ is necessarily equal to the symmetric map of $b$, denoted by $b^{\circ}: Y \times X \rightarrow \overline{\mathbb{R}},(y, x) \mapsto b^{\circ}(y, x)=(x, y)$. Taking two topological vector spaces $X$ and $Y$ in duality, and $b(x, y)=\langle x, y\rangle$, we obtain the classical Legendre-Fenchel transform $B f=f^{*}$.

In the sequel we shall assume that $b(x, y) \in \mathbb{R} \cup\{-\infty\}$ for all $(x, y) \in X \times Y$, and that for all $x \in X$, there exists $y \in Y$ such that $b(x, y) \in \mathbb{R}$, and symmetrically that for all $y \in Y$, there exists $x \in X$ such that $b(x, y) \in \mathbb{R}$.

\section{B. Existence of pre-images}

We shall use the following notion of subdifferentials of Moreau conjugacies (see [15], [16], [17], [18], [19]). Given $f \in \mathscr{F}$ and $y \in Y$, the subdifferential of $f$ at $y$ with respect to $b$ (or $B$ ), denoted by $\partial^{b} f(y)$, or $\partial f(y)$ for brevity, is the set:

$$
\begin{aligned}
& \partial f(y)=\{x \in X \mid b(x, y) \in \mathbb{R}, \\
& \left.\quad b\left(x, y^{\prime}\right)-f\left(y^{\prime}\right) \leq b(x, y)-f(y) \forall y^{\prime} \in Y\right\} .
\end{aligned}
$$

For $g \in \mathscr{G}$ and $x \in X$, the subdifferential of $g$ at $x$ with respect to $b^{\circ}, \partial^{b^{\circ}} g(x)$, will be denoted by $\partial^{\circ} g(x)$ for brevity. When $b(x, y)=\langle x, y\rangle$ we recover the classical definition of subdifferentials.

We shall use the following covering notions. When $\Phi$ is a map from a set $Z$ to the set $\mathscr{P}(W)$ of all subsets of some set $W$, we set $\Phi^{-1}(w)=\{z \in Z \mid w \in \Phi(z)\}$. If $Z^{\prime} \subset Z$ and $W^{\prime} \subset W,\{\Phi(z)\}_{z \in Z^{\prime}}$ is a covering of $W^{\prime}$ if $\cup_{z \in Z^{\prime}} \Phi(z) \supset$ $W^{\prime}$. An element $y \in Z^{\prime}$ is algebraically essential with respect to this covering if $W^{\prime} \not \subset \cup_{z \in Z^{\prime} \backslash\{y\}} \Phi(z)$. When $Z$ is a topological space, $y$ is topologically essential if, for all open neighbourhoods $U$ of $y$ in $Z^{\prime}, W^{\prime} \not \subset \cup_{z \in Z^{\prime} \backslash U} \Phi(z)$. The covering of $W^{\prime}$ by $\{\Phi(z)\}_{z \in Z^{\prime}}$ is algebraically (resp. topologically) minimal if all elements of $Z^{\prime}$ are algebraically (resp. topologically) essential.

The kernel $b$ is said coercive if for all $x \in X$, and all neighbourhoods $V$ of $x$ in $X$, the function

$$
y \in Y \mapsto b_{x, V}(y)=\sup _{z \in V} b(z, y)-b(x, y)
$$

has relatively compact finite sublevel sets, which means that $\left\{y \in Y \mid b_{x, V}(y) \leq \beta\right\}$ is relatively compact for all $\beta \in \mathbb{R}$. We also denote by $\mathscr{F}_{c}$ the set of all $f \in \mathscr{F}$ such that for all $x \in X, y \mapsto b(x, y)-f(y)$ has relatively compact finite superlevel sets, which means that for all $\beta \in \mathbb{R}$, the set $\{y \in Y \mid b(x, y)-f(y) \geq \beta\}$ is relatively compact. For any map $g$ from a topological space $Z$ to $\overline{\mathbb{R}}$, we set: $\operatorname{ldom}(g):=$ $\{z \in Z \mid g(z)<+\infty\}, \operatorname{udom}(g):=\{z \in Z \mid g(z)>$ $-\infty\}, \operatorname{dom}(g):=\operatorname{ldom}(g) \cap \operatorname{udom}(g)$ (the domain of $g$ ), $\operatorname{idom}(g)=\left\{z \in \operatorname{dom}(g) \mid \lim \sup _{z^{\prime} \rightarrow z} g\left(z^{\prime}\right)<+\infty\right\}$.

We shall occasionally need the following assumptions:

(A1) $Y$ is discrete;

$(\mathrm{A} 1)^{\prime} b$ is continuous in the second variable, and $B^{\circ} g(y)>-\infty$ for all $y \in Y$;

(A2) $B^{\circ} g \in \mathscr{F}_{c}$;

(A2) $\quad b$ is coercive and $X^{\prime} \subset \operatorname{idom}(g) \cup g^{-1}(-\infty)$.

(A3) Conditions (A1) or (A1)' and (A2) or (A2)' hold.

Theorem 2.1 ([10, Theorem 3.5]): Let $X^{\prime} \subset X$ and $g \in$ $\mathscr{G}$. Consider the following statements:

(i) Problem $\left(\mathcal{P}^{\prime}\right)$ has a solution,

(ii) $\left\{\left(\partial^{\circ} g\right)^{-1}(y)\right\}_{y \in \operatorname{ldom}\left(B^{\circ} g\right)}$ is a covering of $X^{\prime} \cap u \operatorname{dom}(g)$. We have (ii) $\Rightarrow$ (i) If (A3) is satisfied, then (i) $\Leftrightarrow$ (ii)

\section{Uniqueness of the pre-image}

A map $h$ from a topological space $Z$ to $\overline{\mathbb{R}}$ is quasicontinuous [20] if for all open sets $G$ of $\overline{\mathbb{R}}$, the set $h^{-1}(G)$ is included in the closure of its interior. When $h$ is l.s.c., this is equivalent to the condition that $h$ is the 1.s.c. hull of the upper semicontinuous (u.s.c.) hull of $h$.

Theorem 2.2 ([10, Theorem 4.6]): Let $X^{\prime} \subset X$ and $g \in$ $\mathscr{G}$. Assume that $\left\{\left(\partial^{\circ} g\right)^{-1}(y)\right\}_{y \in \operatorname{ldom}\left(B^{\circ} g\right)}$ is a covering of $X^{\prime} \cap \operatorname{udom}(g)$, and denote by $Z_{a}$ (resp. $Z_{t}$ ) the set of algebraically (resp. topologically) essential elements with respect to this covering. Let $Z=Z_{a} \cup \operatorname{int}\left(Z_{t}\right)$, where $\operatorname{int}\left(Z_{t}\right)$ denotes the interior of $Z_{t}$ relatively to $\operatorname{dom}\left(B^{\circ} g\right)$. Assume that (A3) is satisfied and that $B^{\circ} g$ is quasi-continuous on its domain. Then Problem $\left(\mathcal{P}^{\prime}\right)$ has a solution, and any solution $f$ of $\left(\mathcal{P}^{\prime}\right)$ satisfies

$f \geq B^{\circ} g, \quad$ and $\quad f(y)=B^{\circ} g(y) \quad$ for all $y \in Z$.

Theorem 2.3 ([10, Theorem 4.7]): Let $X^{\prime} \subset X$ and $g \in$ $\mathscr{G}$. Consider the following statements:

(i) Problem $\left(\mathcal{P}^{\prime}\right)$ has a unique solution,

(ii) $\left\{\left(\partial^{\circ} g\right)^{-1}(y)\right\}_{y \in \operatorname{ldom}\left(B^{\circ} g\right)}$ is a topologically minimal covering of $X^{\prime} \cap \operatorname{udom}(g)$.

If (A3) is satisfied, then (i) $\Rightarrow$ (ii) If in addition $B^{\circ} g$ is quasicontinuous on its domain, then (i) $\Leftrightarrow$ (ii)

\section{QUASI-LINEAR FORMS}

We assume now that $Y$ is a Polish (complete separable metric) space. We denote by $\mathbb{R}_{\max }$ the subsemiring of $\overline{\mathbb{R}}_{\max }$ composed of the elements of $\mathbb{R} \cup\{-\infty\}$. The set $\left(\mathbb{R}_{\max }\right)^{Y}$ is a sublattice of $\overline{\mathbb{R}}^{Y}$, which is conditionally complete. We denote by sup or $\vee$ (resp. inf or $\wedge$ ) the supremum (resp. infimum) operation. The set $\left(\mathbb{R}_{\max }\right)^{Y}$ can be endowed with the semimodule structure in which the addition is $(f, g) \mapsto f \vee g$, and the scalar multiplication is $(a, f) \in \mathbb{R}_{\max } \times\left(\mathbb{R}_{\max }\right)^{Y} \mapsto$ $a+f \in\left(\mathbb{R}_{\max }\right)^{Y}$ with $(a+f)(y)=a+f(y)$ for all $y \in Y$. (Semimodules and subsemimodules are defined as modules and submodules over rings [21], [22]). We denote by bd $(Y)$ (resp. $\mathscr{C}(Y)$, resp. $\operatorname{lsc}(Y)$, resp. usc $(Y)$ ) the set of functions from $Y$ to $\mathbb{R}_{\max }$ that are bounded above by a constant (resp. continuous, resp. 1.s.c., resp. u.s.c.). We also use the notations 
$\mathscr{C}_{\mathrm{b}}(Y):=\mathscr{C}(Y) \cap \operatorname{bd}(Y)$ and $\operatorname{usc}_{\mathrm{b}}(Y):=\operatorname{usc}(Y) \cap \operatorname{bd}(Y)$. All these sets are subsemimodules of $\left(\mathbb{R}_{\max }\right)^{Y}$.

Definition 3.1: Let $\mathscr{M}$ be a subsemimodule of $\left(\mathbb{R}_{\max }\right)^{Y}$. A map $F: \mathscr{M} \rightarrow \mathbb{R}_{\max }$ (or $\overline{\mathbb{R}}_{\max }$ ) is a quasi- (max-plus) -linear form (on $\mathscr{M}$ ) if it is isotone, that is

$$
\varphi \leq \psi \Longrightarrow F(\varphi) \leq F(\psi) \text { for all } \varphi, \psi \in \mathscr{M},
$$

if it is additively homogeneous, that is

$$
F(\lambda+\varphi)=\lambda+F(\varphi) \text { for all } \lambda \in \mathbb{R}_{\max }, \varphi \in \mathscr{M},
$$

and if there exists $\alpha \in \mathbb{R}_{\max }$ such that

$$
F(\varphi \vee \psi) \leq \alpha+F(\varphi) \vee F(\psi) \text { for all } \varphi, \psi \in \mathscr{M} .
$$

A quasi-linear form $F$ on $\mathscr{M}$ is continuous if it preserves nondecreasing converging sequences.

We denote by $\rho(F)$ the infimum of the $\alpha$ satisfying (2C), and by $\mathscr{Q} \mathscr{L}(\mathscr{M})$ the set of continuous quasi-linear forms from $\mathscr{M}$ to $\mathbb{R}_{\max }$. When $F$ takes at least one value in $\mathbb{R}$, $\rho(F) \geq 0$ and one can take $\alpha=\rho(F)$ in (2c). Otherwise $\rho(F)=-\infty$.

A map $F: \mathscr{M} \rightarrow \overline{\mathbb{R}}_{\max }$ is a quasi-linear form such that $\rho(F) \leq 0$ if, and only if, $F$ is a max-plus linear form, that is $F$ satisfies (2b and $F(\varphi \vee \psi)=F(\varphi) \vee F(\psi)$ for all $\varphi, \psi \in \mathscr{M}$. We set $\mathscr{L}(\mathscr{M}):=\{F \in \mathscr{Q} \mathscr{L}(\mathscr{M}) \mid \rho(F) \leq 0\}$. Given $f: Y \rightarrow \overline{\mathbb{R}}$, the map $F:\left(\mathbb{R}_{\max }\right)^{Y} \rightarrow \overline{\mathbb{R}}_{\max }$ defined by

$$
F(\varphi)=\sup _{y \in Y}(\varphi(y)-f(y)) \text { for all } \varphi \in\left(\mathbb{R}_{\max }\right)^{Y}
$$

is a continuous (as a quasi-linear form) max-plus linear form on $\left(\mathbb{R}_{\max }\right)^{Y}$ and so on $\mathscr{C}_{\mathrm{b}}(Y)$. A map $f$ satisfying (3) is called a density of $F$. Conversely, since $Y$ is a separable metric space, any element of $\mathscr{L}\left(\mathscr{C}_{\mathrm{b}}(Y)\right)$ has a unique 1.s.c. density [8, Th. 4.8 and Cor. 3.12] (see also [23], [24], [7]), which is bounded below by some real constant. Note however that a max-plus linear form is not necessarily continuous.

Let $\mu$ be a finite positive measure on $Y$, let $\varepsilon>0$ and consider the map $F: \mathscr{C}_{\mathrm{b}}(Y) \rightarrow \mathbb{R}_{\max }$ with

$$
F(\varphi)=\varepsilon \log \left(\int_{Y} \exp \left(\frac{\varphi(y)}{\varepsilon}\right) d \mu(y)\right),
$$

for all $\varphi \in \mathscr{C}_{\mathrm{b}}(Y)$. Then $F$ is a continuous quasi-linear form with $\rho(F) \leq \varepsilon \log (2)$. The maps $F$ of the form (4) where $\mu$ is a probability measure occur in large deviations principles. We shall denote by $\mathscr{L}_{\mathcal{D}}(Y)$ the set of all such maps.

Let $F_{i} \in \mathscr{Q} \mathscr{L}(\mathscr{M})$, for $i \in I$, such that $\sup _{i \in I} \rho\left(F_{i}\right)<$ $+\infty$. Then the map $\sup _{i \in I} F_{i}: \mathscr{M} \rightarrow \overline{\mathbb{R}}_{\max }, \varphi \mapsto$ $\sup _{i \in I} F_{i}(\varphi)$ is a continuous quasi-linear form on $\mathscr{M}$ and it satisfies $\rho\left(\sup _{i \in I} F_{i}\right) \leq \sup _{i \in I} \rho\left(F_{i}\right)$.

Proposition 3.2: Any $F \in \mathscr{Q} \mathscr{L}\left(\mathscr{C}_{\mathrm{b}}(Y)\right)$ admits a unique extension to a continuous quasi-linear form on $\operatorname{lsc}(Y)$ (with values in $\overline{\mathbb{R}}_{\max }$ ) that we also denote by $F$ :

$$
F(\varphi)=\sup _{\psi \in \mathscr{C}_{\mathrm{b}}(Y), \psi \leq \varphi} F(\psi) \quad \text { for all } \varphi \in \operatorname{lsc}(Y),
$$

and a maximal extension to a continuous quasi-linear form on $\left(\mathbb{R}_{\max }\right)^{Y}$ that we also denote by $F$ :

$$
F(\varphi)=\inf _{\psi \in \operatorname{lsc}(Y), \psi \geq \varphi} F(\psi) \quad \text { for all } \varphi \in\left(\mathbb{R}_{\max }\right)^{Y} .
$$

The value of $\rho(F)$ for the maximal extension of $F$ to $\left(\mathbb{R}_{\max }\right)^{Y}$ and for its restriction to $\mathscr{C}_{\mathrm{b}}(Y)$ coincide.

If $A$ is a subset of $Y$, we denote by $\mathbf{1}_{A}: Y \rightarrow \mathbb{R}_{\max }$ the max-plus characteristic function of $A: \mathbf{1}_{A}(y)=0$ if $y \in A$ and $\mathbf{1}_{A}(y)=-\infty$ otherwise. If $F$ is as in Proposition 3.2 we shall also denote by $F$ the map $F: \mathcal{P}(Y) \rightarrow \mathbb{R}_{\max }, \quad A \mapsto$ $F\left(\mathbf{1}_{A}\right)$. This map is isotone: $A \subset B \Longrightarrow F(A) \leq F(B)$, and it satisfies some inner and outer-continuity properties. If $F$ is a continuous max-plus linear form or is an element of $\mathscr{L} \mathscr{D}_{\varepsilon}(Y)$, with $\varepsilon>0$, then the exponential of its restriction to $\mathcal{P}(Y)$ is a capacity in the sense defined in [25], [26]. Some other related sets of functions on $\mathcal{P}(Y)$ are defined in [27], [28].

\section{WEAK CONVERGENCE OF QUASI-LINEAR FORMS}

Let $F \in \mathscr{Q} \mathscr{L}\left(\mathscr{C}_{\mathrm{b}}(Y)\right)$ and $\left(F_{n}\right)_{n \in \mathbb{N}}$ be a sequence of $\mathscr{Q} \mathscr{L}\left(\mathscr{C}_{\mathrm{b}}(Y)\right)$. We say that $F_{n}$ weakly converges towards $F$ if $\lim _{n \rightarrow \infty} F_{n}(\varphi)=F(\varphi)$ for all $\varphi \in \mathscr{C}_{\mathrm{b}}(Y)$. In that case, we get that $\rho(F) \leq \liminf _{n \rightarrow \infty} \rho\left(F_{n}\right)$. When $F_{n} \in \mathscr{L}_{\varepsilon}(Y)$ is defined from the measure $\mu_{n}$, then $F \in \mathscr{L} \mathscr{D}_{\varepsilon}(Y)$ and the weak convergence of $F_{n}$ towards $F$ is equivalent to the weak (or narrow) convergence of $\mu_{n}$ towards the measure $\mu$ corresponding to $F$. If all the $F_{n}$ are continuous maxplus linear forms with 1.s.c. densities $f_{n}$, then $F$ is also a continuous max-plus linear form and the weak convergence of $F_{n}$ towards $F$ is equivalent to the weak convergence of the cost measure with density $f_{n}$ towards the cost measure with density $f$ (see [29]), where $f$ is the 1.s.c. density of $F$.

We say that an element $F$ of $\mathscr{Q} \mathscr{L}\left(\mathscr{C}_{\mathrm{b}}(Y)\right)$ is tight if $\inf _{K \subset Y, K \text { compact }} F\left(K^{c}\right)=-\infty$. A sequence $\left(F_{n}\right)_{n \in \mathbb{N}}$ of $\mathscr{Q} \mathscr{L}\left(\mathscr{C}_{\mathrm{b}}(Y)\right)$ is asymptotically tight if $\lim _{\sup _{n \rightarrow \infty}} \rho\left(F_{n}\right)<$ $+\infty$ and

$$
\inf _{K \subset Y, K \text { compact }} \limsup _{n \rightarrow \infty} F_{n}\left(K^{c}\right)=-\infty .
$$

Since $Y$ is a Polish space, any element of $\mathscr{L}_{\varepsilon}(Y)$, with $\varepsilon>0$, is tight. An element of $\mathscr{L}\left(\mathscr{C}_{\mathrm{b}}(Y)\right)$ is tight if, and only if, its 1.s.c. density $f$ is inf-compact, that is $\{y \in Y \mid$ $f(y) \leq \alpha\}$ is compact for all $\alpha \in \mathbb{R}$.

Theorem 4.1: Let $F \in \mathscr{Q} \mathscr{L}\left(\mathscr{C}_{\mathrm{b}}(Y)\right)$ and $\left(F_{n}\right)_{n \in \mathbb{N}}$ be a sequence of $\mathscr{Q} \mathscr{L}\left(\mathscr{C}_{\mathrm{b}}(Y)\right)$. Denote also by $F$ and $F_{n}$ the extensions given by Proposition 3.2 Consider the following statements:

$$
\begin{aligned}
& F_{n} \text { weakly converges towards } F, \\
& \liminf _{n \rightarrow \infty} F_{n}(\varphi) \geq F(\varphi) \text { for all } \varphi \in \operatorname{lsc}(Y), \\
& \limsup _{n \rightarrow \infty} F_{n}(\varphi) \leq F(\varphi) \text { for all } \varphi \in \operatorname{usc}_{\mathrm{b}}(Y), \\
& \liminf _{n \rightarrow \infty} F_{n}(G) \geq F(G) \text { for all open } G \subset Y, \\
& \limsup _{n \rightarrow \infty} F_{n}(C) \leq F(C) \text { for all closed } C \subset Y, \\
& \limsup _{n \rightarrow \infty} F_{n}(K) \leq F(K) \text { for all compact } K \subset Y .
\end{aligned}
$$

We have $677 \Rightarrow(5) \Rightarrow(6) \Rightarrow(8), 77 \Rightarrow(9) \Rightarrow(10)$. If $\rho(F) \leq$ $\lim _{n \rightarrow \infty} \rho\left(F_{n}\right)=0$, then $\left[5 \Leftrightarrow 677 \Leftrightarrow\right.$ 89. If $\left(F_{n}\right)_{n \in \mathbb{N}}$ is asymptotically tight then $9 \Leftrightarrow 10$.

When $F_{n} \in \mathscr{L} \mathscr{D}_{\varepsilon_{n}}(Y)$ with corresponding probability measure $\mu_{n}, F$ is a continuous max-plus linear form with 
density $f$, and $\lim _{n \rightarrow \infty} \varepsilon_{n}=0,\left(\mu_{n}\right)_{n \in \mathbb{N}}$ obeys the large deviation principle of Varadhan [30] with rate function $f$ if, and only if, $f$ is nonnegative and inf-compact and 89 holds. In this context, the implication $899 \Rightarrow 5$ is called the contraction principle of Varadhan, and some other implications in Theorem 4.1 are proved in [30] and in [31, Theorem 3.1.3] (see also [13] and [32]). In the context of capacities, the conditions 8110 define the vague convergence and the conditions 89 define the narrow (weak) convergence [25]. In the context of continuous max-plus linear forms, some of the implications in Theorem 4.1 are proved in [29]. The following result is also classical for large deviations. It was stated for max-plus linear forms in [29].

Theorem 4.2: Let $\left(F_{n}\right)_{n \in \mathbb{N}}$ be a sequence of $\mathscr{Q L}\left(\mathscr{C}_{\mathrm{b}}(Y)\right)$ such that $\limsup _{n \rightarrow \infty} F_{n}(Y)<+\infty$ and $\lim _{n \rightarrow \infty} \rho\left(F_{n}\right)=0$. Then there exists $F \in \mathscr{L}\left(\mathscr{C}_{\mathrm{b}}(Y)\right)$ and a subsequence of $\left(F_{n}\right)_{n \in \mathbb{N}}$ such that 810 holds for that subsequence.

\section{UNIQUENESS OF PRE-IMAGES OF MOREAU CONJUGACIES AND CONVERGENCE OF QUASI-LINEAR FORMS}

Let $X, Y, B, B^{\circ}, b, b^{\circ}$ be as in Section

We say that $b$ is strongly coercive if for all $x \in X$ and all neighbourhoods $V$ of $x$ in $X$, there exists a finite subset $W$ of $V$ such that the function $b_{x, W}$ defined as in (1) has relatively compact finite sublevel sets.

We say that $b$ is upper (strongly) coercive if for all $x \in X$, and all neighbourhoods $V$ of $x$ in $X$, there exists a finite subset $W$ of $V$ such that $b(x, \cdot)$ is bounded above on each finite sublevel set of $b_{x, W}$.

If $b$ is strongly coercive and continuous in the second variable, then $b$ is coercive and upper coercive. If $b(x, y)=$ $\langle x, y\rangle$, then $b$ is upper coercive (take $W=\{t x\}$ with $t>1$ near enough from 1). If in addition $X=Y=\mathbb{R}^{n}$, then $b$ is strongly coercive (take $W=\left\{x \pm \varepsilon e_{i} \mid 1 \leq i \leq n\right\}$ with $\varepsilon>0$ small enough, and $\left(e_{1}, \ldots, e_{n}\right)$ a basis of $\left.\mathbb{R}^{n}\right)$.

The following result motivates the study of Problem $\left(\mathcal{P}^{\prime}\right)$.

Theorem 5.1: Let $\left(F_{n}\right)_{n \in \mathbb{N}}$ be a sequence of $\mathscr{Q L}\left(\mathscr{C}_{\mathrm{b}}(Y)\right)$ such that $\lim _{n \rightarrow \infty} \rho\left(F_{n}\right)=0$. Assume that $F_{n}$ weakly converges towards $F \in \mathscr{L}\left(\mathscr{C}_{\mathrm{b}}(Y)\right)$, with 1.s.c. density $f$, and that $b$ is continuous in the second variable and upper coercive. Let $g: X \rightarrow \overline{\mathbb{R}}$ be defined by:

$$
g(x)=\limsup _{n \rightarrow \infty} F_{n}(b(x, \cdot)) \quad \text { for all } x \in X .
$$

Then

$$
B f \leq g \text { and } B f=g \text { on } \operatorname{idom}(g) \cup g^{-1}(-\infty) .
$$

The next result follows from Theorems 4.14 .25 .12 .1 2.2 and 2.3 It needs the following technical assumption:

(A4) Conditions (A1) or (A1)' hold; Condition (A2) holds or $b$ is coercive; $b$ is upper coercive; and $B^{\circ} g$ is quasi-continuous on its domain.

Theorem 5.2: Let $\left(F_{n}\right)_{n \in \mathbb{N}}$ be an asymptotically tight sequence of $\mathscr{Q} \mathscr{L}\left(\mathscr{C}_{\mathrm{b}}(Y)\right)$, such that $\limsup _{n \rightarrow \infty} F_{n}(Y)<$ $+\infty$ and $\lim _{n \rightarrow \infty} \rho\left(F_{n}\right)=0$. Let $g: X \rightarrow \mathbb{\mathbb { R }}$ be defined by 11 and denote by $\bar{F}$ the continuous max-plus linear form with density $B^{\circ} g$. Assume that (A4) is satisfied. Then

(i) There exists $F \in \mathscr{L}\left(\mathscr{C}_{\mathrm{b}}(Y)\right)$, and a subsequence of $\left(F_{n}\right)_{n \in \mathbb{N}}$ which converges weakly towards $F$.

(ii) $\left\{\left(\partial^{\circ} g\right)^{-1}(y)\right\}_{y \in \operatorname{ldom}\left(B^{\circ} g\right)}$ is a covering of $\operatorname{idom}(g)$.

(iii) If $F$ is an accumulation point of $\left(F_{n}\right)_{n \in \mathbb{N}}$ for the weak convergence, and if $f$ is the 1.s.c. density of $F$, then $f \geq$ $B^{\circ} g$. Hence $\lim \sup _{n \rightarrow \infty} F_{n}(C) \leq \bar{F}(C)$ for all closed $C \subset$ $Y$.

Assume in addition that the limsup in (11) is a limit, and let $Z$ be defined as in Theorem 2.2 with $X^{\prime}=\operatorname{idom}(g)$.

(iv) If $F$ and $f$ are as in (iii) then $f=B^{\circ} g$ on $Z$. Hence $\liminf _{n \rightarrow \infty} F_{n}(G) \geq \bar{F}(G \cap Z)$ for all open $G \subset Y$.

(v) If $\left\{\left(\partial^{\circ} g\right)^{-1}(y)\right\}_{y \in \operatorname{ldom}\left(B^{\circ} g\right)}$ is a topologically minimal covering of idom $(g)$, then $F_{n}$ weakly converges towards $\bar{F}$.

The following result can be used to obtain the "compactness" of the sequence $\left(F_{n}\right)_{n \in \mathbb{N}}$.

Proposition 5.3: Let $\left(F_{n}\right)_{n \in \mathbb{N}}$ be a sequence of $\mathscr{Q} \mathscr{L}\left(\mathscr{C}_{\mathrm{b}}(Y)\right)$ such that $\lim _{n \rightarrow \infty} \rho\left(F_{n}\right)=0$, and let $g: X \rightarrow \overline{\mathbb{R}}$ be given by 11. Assume that $b$ is strongly coercive, and that there exists $x_{0} \in \operatorname{idom}(g)$ such that $b\left(x_{0}, \cdot\right)$ is bounded below by some real constant. Then $\limsup _{n \rightarrow \infty} F_{n}(Y)<+\infty$ and $\left(F_{n}\right)_{n \in \mathbb{N}}$ is asymptotically tight.

Corollary 5.4 (Generalised Gärtner-Ellis theorem):

Let $\left(F_{n}\right)_{n \in \mathbb{N}}$ be a sequence of $\mathscr{Q} \mathscr{L}\left(\mathscr{C}_{\mathrm{b}}(Y)\right)$ such that $\lim _{n \rightarrow \infty} \rho\left(F_{n}\right)=0$, let $g: X \rightarrow \overline{\mathbb{R}}$ be given by (11), and assume that the limsup there is a limit. Assume that (A1) or (A1)' hold, that $b$ is strongly coercive, that $B^{\circ} g$ is quasi-continuous on its domain and that there exists $x_{0} \in \operatorname{idom}(g)$ such that $b\left(x_{0}, \cdot\right)$ is lower bounded by some real constant. Then the conclusions of Theorem 5.2 hold.

When $B$ is the Legendre-Fenchel transform on $\mathbb{R}^{n}, x_{0}=$ $0, F_{n} \in \mathscr{L} \mathscr{D}_{\varepsilon_{n}}(Y)$ with $\lim _{n \rightarrow \infty} \varepsilon_{n}=0$, the statement of Corollary 5.4 contains the Gärtner-Ellis theorem as stated in [13, Th. 2.3.6]. Indeed, $b(0, \cdot) \equiv 0, b$ is strongly coercive (see above), $B^{\circ} \mathrm{g}$ is quasi-continuous on its domain (see [10, Lemma 6.1]), $B^{\circ} g(y)>-\infty$ for all $y \in Y$ when $g$ is proper, in particular when idom $(g) \neq \emptyset$. Moreover, by [10, Proposition 6.3 and Corollary 6.4], $\left\{\left(\partial^{\circ} g\right)^{-1}(y)\right\}_{y \in \operatorname{ldom}\left(B^{\circ} g\right)}$ is a topologically minimal covering of $\operatorname{idom}(g)$, when $g$ is an essential smooth 1.s.c. proper convex function on $\mathbb{R}^{n}$, which means that the interior of its domain $\operatorname{idom}(g)$ is nonempty, that $g$ is differentiable in idom $(g)$, and that the norm of the differential of $g$ at $x$ tends to infinity when $x$ goes to the boundary of $\operatorname{dom}(g)$, see [33, Section 26].

The proof of our generalisation of the Gärtner-Ellis theorem essentially uses compactness arguments together with the uniqueness of the pre-image of an essential smooth convex function by the Legendre-Fenchel transform. This last argument was made explicit by O'Brien and Vervaat [26, Theorem 4.1 (c)], and Puhalskii [34, Lemmas 3.2 and 3.5] for the Gärtner-Ellis theorem, and by Gulinsky [35, Theorems 4.7 and 5.3] for the more general case where $B$ is the LegendreFenchel transform, and $F_{n}(\varphi)=\varepsilon_{n} \log J_{n}\left(\exp \left(\frac{\varphi}{\varepsilon_{n}}\right)\right)$ with $J_{n}(\varphi \vee \psi) \leq J_{n}(\varphi)+J_{n}(\psi)$. 
If $F_{n}$ weakly converges towards $F$ with a density $f$ which is not essentially strictly convex (or equivalently such that its Legendre-Fenchel transform $f^{*}$ is not essentially smooth) the Gärtner-Ellis theorem only gives the inequalities of Assertions (iii) and (iv) of Theorem 5.2 with $Z \neq Y$, thus the rate function $f$ cannot be identified. The classical method is to adapt the proof of the Gärtner-Ellis theorem, whereas using Theorem 5.2 one may simply consider a different kernel $b$ than that of the Legendre-Fenchel transform. Moreover, Proposition 5.3 can be applied to another kernel.

The following result is useful in the study of optimal control problems.

Theorem 5.5: Assume that, for $i \in I,\left(F_{n, i}\right)_{n \in \mathbb{N}}$ is an asymptotically tight sequence of $\mathscr{Q} \mathscr{L}\left(\mathscr{C}_{\mathrm{b}}(Y)\right)$, such that $\lim \sup _{n \rightarrow \infty} F_{n, i}(Y)<\infty$ and $\lim _{n \rightarrow \infty} \rho\left(F_{n, i}\right)=0$. Let $g: X \rightarrow \overrightarrow{\mathbb{R}}$ be defined by

$$
g(x)=\sup _{i \in I} \limsup _{n \rightarrow \infty} F_{n, i}(b(x, \cdot)) \quad \text { for all } x \in X,
$$

and denote by $\bar{F}$ the continuous max-plus linear form with density $B^{\circ} \mathrm{g}$. Assume that (A4) is satisfied. Then

(i) There exists $F \in \mathscr{L}\left(\mathscr{C}_{\mathrm{b}}(Y)\right)$ such that $\sup _{i \in I} \lim \sup _{n \rightarrow \infty} F_{n, i}(G) \geq F(G)$ for all open $G \subset Y$, and $\sup _{i \in I} \limsup \sup _{n \rightarrow \infty} F_{n, i}(C) \leq F(C)$ for all closed $C \subset Y$.

(ii) The 1.s.c. density $f$ of $F$ satisfies (12). Hence $\left\{\left(\partial^{\circ} g\right)^{-1}(y)\right\}_{y \in \operatorname{ldom}\left(B^{\circ} g\right)}$ is a covering of $\operatorname{idom}(g)$.

(iii) We have $f \geq B^{\circ} g$, hence, for all closed $C \subset Y$, $\sup _{i \in I} \lim \sup _{n \rightarrow \infty} F_{n, i}(C) \leq \bar{F}(C)$.

(iv) Let $Z$ be defined as in Theorem 2.2 for $X^{\prime}=\operatorname{idom}(g)$. Then $f=B^{\circ} g$ on $Z$. Hence, for all open $G \subset Y$, $\sup _{i \in I} \lim \sup _{n \rightarrow \infty} F_{n, i}(G) \geq \bar{F}(G \cap Z)$.

\section{AN APPLICATION TO THE OPTIMAL LONG-TERM RATE OF AN INVESTMENT MODEL}

We consider here the simple Merton model [36] of an investor who has the possibility to invest in one bank account paying a fixed interest rate $r>0$ and in one stock or risky asset whose price is a log-normal diffusion with expected rate $\alpha>r$ and rate variation $\sigma$, and who has the ability to transfer funds between the assets with no cost. We denote by $W_{t}$ the total wealth of the investor at time $t$, and by $\xi_{t}$ the proportion of fund invested in the risky asset. The process $W_{t}$ satisfies the following stochastic differential equation:

$$
d W_{t}=\left(r+(\alpha-r) \xi_{t}\right) W_{t} d t+\sigma \xi_{t} W_{t} d B_{t}
$$

where $B_{t}$ is a Brownian motion. The control process $\xi=$ $\left(\xi_{t}\right)_{t \geq 0}$ is supposed to be adapted to the Brownian filtration and stationary. We allow borrowing and shortselling, which means that $\xi_{t}$ can be any real number. One is interested in maximising some function of the long term growth rate of the investor. One possibility is to consider the risk-sensitive problem

$$
\sup _{\xi} \limsup _{T \rightarrow \infty} \frac{1}{T(1-\gamma)} \log E\left[\left(W_{T}\right)^{1-\gamma}\right]
$$

where $E$ denotes the expectation and $\gamma$ is the risk-aversion coefficient. Another possibility is to consider, for $c \in \mathbb{R}$ :

$$
\sup _{\xi} \limsup _{T \rightarrow \infty} \frac{1}{T} \log P\left[\left(\log \left(W_{T}\right) / T \geq c\right] .\right.
$$

In [14] the latter problem was considered for a different investment model and the relation with the risk-sensitive problems with $\gamma<1$ was discussed and used to obtain a result of the same nature as the Gärtner-Ellis theorem.

We apply here the results of the previous sections to compute the quantity (13). Let $Y=X=\mathbb{R}$ and consider the quasi-linear form $F_{W_{0}, T, \xi}$ on $\mathscr{C}_{\mathrm{b}}(Y)$ defined by

$$
F_{W_{0}, T, \xi}(\varphi)=\frac{1}{T} \log E\left[\exp \left(T \varphi\left(\log \left(W_{T}\right) / T\right)\right) \mid W_{0}\right]
$$

and extended as in Proposition 3.2 together with the quasi-linear form $F_{W_{0}, T}=\sup _{\xi} F_{W_{0}, T, \xi}$. Then, for all $W_{0}, T, \xi, F_{W_{0}, T, \xi}(Y)=F_{W_{0}, T}(Y)=0$, and $F_{W_{0}, T, \xi}$ and $F_{W_{0}, T} \in \mathscr{Q} \mathscr{L}\left(\mathscr{C}_{\mathrm{b}}(Y)\right)$. Moreover $\rho\left(F_{W_{0}, T, \xi}\right) \leq \log (2) / T$, thus $\lim _{T \rightarrow \infty} \rho\left(F_{W_{0}, T, \xi}\right)=\lim _{T \rightarrow \infty} \rho\left(F_{W_{0}, T}\right)=0$.

Let $b(x, y)=x y$ be the kernel of the LegendreFenchel transform. Then for all $x \in \mathbb{R}, F_{W_{0}, T, \xi}(b(x, \cdot))=$ $\frac{1}{T} \log E\left[\left(W_{T}\right)^{x} \mid W_{0}\right]$ is a risk-sensitive utility function. We have the homogeneity property: $F_{W_{0}, T, \xi}(b(x, \cdot))=$ $\frac{x \log \left(W_{0}\right)}{T}+F_{1, T, \xi}(b(x, \cdot))$. Let $g: X \rightarrow \overline{\mathbb{R}}$ be defined by

$$
g(x)=\sup _{\xi \in \mathbb{R}} x\left(r+(\alpha-r) \xi+(x-1) \frac{\sigma^{2} \xi^{2}}{2}\right)
$$

for $x \in X$. Then $g(x)=x\left(r+\frac{(\alpha-r)^{2}}{2 \sigma^{2}(1-x)}\right)$ if $0 \leq x<1$ and $g(x)=+\infty$ otherwise. Moreover, for $0 \leq x<1$, the proportion $\bar{\xi}_{x}=\frac{\alpha-r}{\sigma^{2}(1-x)}$ realises the maximum in (14). We have, for all $T>0$ and $x \in X, F_{1, T}(b(x, \cdot))=g(x)$, and for all $T>0, W_{0}>0$ and $0 \leq x<1$, the constant control process $\xi_{t} \equiv \bar{\xi}_{x}$ maximises $F_{W_{0}, T, \xi}(b(x, \cdot))$. Hence

$$
\begin{aligned}
& \sup _{\xi} \limsup _{T \rightarrow \infty} F_{W_{0}, T, \xi}(b(x, \cdot)) \\
& \quad=\lim _{T \rightarrow \infty} F_{W_{0}, T}(b(x, \cdot))=g(x) .
\end{aligned}
$$

The Legendre-Fenchel transform $g^{*}$ of $\mathrm{g}$ is given by $g^{*}(y)=$ $\left(\sqrt{y-r}-\frac{\alpha-r}{\sqrt{2} \sigma}\right)^{2}$ if $y \geq z_{0}:=r+\frac{(\alpha-r)^{2}}{2 \sigma^{2}}$ and $g^{*}(y)=0$ otherwise. So, if $\bar{F}$ and $Z$ are defined as in Theorem 5.2 we get $Z=\left(z_{0},+\infty\right)$, and $\bar{F}((c,+\infty) \cap Z)=\bar{F}([c,+\infty))=$ $-g^{*}(c)$ for all $c \in \mathbb{R}$. If, for any sequence $T_{n}$ going to infinity, the sequence $\left(F_{W_{0}, T_{n}}\right)_{n \geq 0}$ were asymptotically tight, then Theorem 5.2 would show:

$$
\begin{aligned}
& \liminf _{T \rightarrow+\infty} F_{W_{0}, T}(G) \geq \bar{F}(G \cap Z) \text { for all open } G \subset \mathbb{R}, \\
& \limsup _{T \rightarrow+\infty} F_{W_{0}, T}(C) \leq \bar{F}(C) \text { for all closed } C \subset \mathbb{R} .
\end{aligned}
$$

In particular this would show:

$$
\begin{array}{r}
\lim _{T \rightarrow+\infty} \sup _{\xi} \frac{1}{T} \log P\left[\left(\log \left(W_{T}\right) / T \geq c \mid W_{0}\right]\right. \\
=\lim _{T \rightarrow+\infty} F_{W_{0}, T}([c,+\infty))=-g^{*}(c) .
\end{array}
$$

However, since $0 \notin \operatorname{idom}(g)$, one cannot use Proposition 5.3 to show the asymptotic tightness of $\left(F_{W_{0}, T_{n}}\right)_{n \geq 0}$. 
Let us thus replace the process $\log \left(W_{T}\right) / T$ by its maximum with some constant $a<z_{0}$. This amounts to replacing $F_{W_{0}, T, \xi}$ by the quasi-linear form $G_{W_{0}, T, \xi}(\varphi):=$ $F_{W_{0}, T, \xi}\left(\varphi \circ \chi_{a}\right)$ where $\chi_{a}(x)=x \vee a$. We also consider $G_{W_{0}, T}=\sup _{\xi} G_{W_{0}, T, \xi}$. We take now $Y=[a,+\infty)$ and $X=[0,+\infty)$. The kernel $b(x, y)=x y$ is strongly coercive with respect to these new sets $X$ and $Y$. The corresponding Moreau conjugacies $B$ and $B^{\circ}$ are the Legendre-Fenchel transform composed with the restriction operation to $Y$ and $X$ respectively. Since $\rho\left(F_{W_{0}, T, \xi}\right)$ tends to 0 when $T$ goes to infinity, and $b\left(x, \chi_{a}(y)\right)=b(x, y) \vee x a$ for all $x \in$ $[0,+\infty)$ and $y \in \mathbb{R}$, we get that $\lim _{T \rightarrow \infty} G_{W_{0}, T}(b(x, \cdot))=$ $\lim _{T \rightarrow \infty} F_{W_{0}, T}(b(x, \cdot)) \vee x a=g(x)$ for all $x \in X$. Moreover, $B^{\circ} g$ is the restriction of $g^{*}$ to $Y$. With respect to the new set $Y, b(x, \cdot)$ is lower bounded for all $x \in X$ and $\operatorname{idom}(g)=[0,1)$, hence Proposition 5.3 shows that $G_{W_{0}, T_{n}}$ is asymptotically tight for any sequence $T_{n}$ tending to infinity. Then the conclusions (16) of Theorem 5.2 hold with $F_{W_{0}, T}$ replaced by $G_{W_{0}, T}$, and with $Z=\left(z_{0},+\infty\right)$ unchanged. Since $\mathbf{1}_{A} \circ \chi_{a}=\mathbf{1}_{A}$ if $a \notin A$, and $a$ can be chosen small enough, we deduce (17).

Let us now apply Theorem 5.5 to the sequences $\left(G_{W_{0}, T_{n}, \xi}\right)_{n \in \mathbb{N}}$, with $T_{n}$ tending to infinity, and $W_{0}$ fixed, and where the parameter $i$ corresponds to the couple composed of the control process $\xi$ and of the sequence $\left(T_{n}\right)_{n \geq 0}$. We obtain, by the same arguments as before, that for all $c \in \mathbb{R}$ :

$$
\begin{aligned}
& \quad \sup _{\xi} \limsup _{T \rightarrow+\infty} \frac{1}{T} \log P\left[\left(\log \left(W_{T}\right) / T \geq c \mid W_{0}\right]\right. \\
& \quad=\sup _{\xi} \limsup _{T \rightarrow+\infty} F_{W_{0}, T, \xi}([c,+\infty))=-g^{*}(c) .
\end{aligned}
$$

The latter conclusion is of the same nature as the one of [14, Theorem 3.1]. Note however that for the proof of (18) one does not need that the supremum in 15 is attained and that for this maximum the limsup is a limit, as is required in [14], even if these properties hold in our example. However, these conditions were useful to prove (17), and so to prove that in (18) the sup and limsup operations commute.

\section{REFERENCES}

[1] J.-J. Moreau, "Inf-convolution, sous-additivité, convexité des fonctions numériques," J. Math. Pures Appl. (9), vol. 49, pp. 109-154, 1970.

[2] R. T. Rockafellar and R. J.-B. Wets, Variational analysis. Berlin: Springer-Verlag, 1998.

[3] I. Singer, Abstract convex analysis. New York: John Wiley \& Sons Inc., 1997.

[4] R. A. Cuninghame-Green, Minimax Algebra, ser. Lecture notes in Economics and Mathematical Systems. Springer, 1979, no. 166.

[5] V. Maslov and S. Samborskiı̆, Eds., Idempotent analysis, ser. Adv. in Sov. Math. RI: AMS, 1992, vol. 13.

[6] F. Baccelli, G. Cohen, G. J. Olsder, and J.-P. Quadrat, Synchronization and linearity : an algebra for discrete events systems. New-York: John Wiley \& Sons, 1992.

[7] V. Kolokoltsov and V. Maslov, Idempotent analysis and applications. Kluwer Acad. Publisher, 1997.

[8] M. Akian, "Densities of idempotent measures and large deviations," Transactions of the American Mathematical Society, vol. 351, no. 11, pp. 4515-4543, 1999.

[9] M. Gondran and M. Minoux, Graphes, diö̈des et semi-anneaux. Paris: TEC \& DOC, 2001.

[10] M. Akian, S. Gaubert, and V. N. Kolokoltsov, Set coverings and invertibility of functional Galois connections, ser. Contemporary Mathematics. Providence, RI: Amer. Math. Soc., 2005, vol. 377, pp. 19-51.
[11] N. N. Vorobyev, "Extremal algebra of positive matrices," Elektron. Informationsverarbeitung und Kybernetik, vol. 3, pp. 39-71, 1967, in Russian.

[12] K. Zimmermann, Extremální Algebra. Praha: Ekonomický ùstav C̆SAV, 1976, (in Czech).

[13] A. Dembo and O. Zeitouni, Large deviations techniques and applications. Boston, MA: Jones and Barlett, 1993.

[14] H. Pham, "A large deviations approach to optimal long term investment," Finance Stoch., vol. 7, no. 2, pp. 169-195, 2003.

[15] E. J. Balder, "An extension of duality-stability relations to nonconvex optimization problems," SIAM J. Control Optimization, vol. 15, no. 2, pp. 329-343, 1977.

[16] S. Dolecki and S. Kurcyusz, "On $\Phi$-convexity in extremal problems," SIAM J. Control Optimization, vol. 16, no. 2, pp. 277-300, 1978.

[17] P. O. Lindberg, "A generalization of Fenchel conjugation giving generalized Lagrangians and symmetric nonconvex duality," in Survey of mathematical programming (Proc. Ninth Internat. Math. Programming Sympos., Budapest, 1976), Vol. 1. Amsterdam: North-Holland, 1979, pp. 249-267.

[18] J.-E. Martínez-Legaz, "Quasiconvex duality theory by generalized conjugation methods," Optimization, vol. 19, no. 5, pp. 603-652, 1988.

[19] J.-E. Martínez-Legaz and I. Singer, "Subdifferentials with respect to dualities," ZOR-Math. Methods Oper. Res., vol. 42, no. 1, pp. 109$125,1995$.

[20] T. Neubrunn, "Quasi-continuity," Real Anal. Exchange, vol. 14, no. 2, pp. $259-306,1988 / 89$.

[21] G. L. Litvinov, V. P. Maslov, and G. B. Shpiz, "Idempotent functional analysis: An algebraical approach," Mat. Notes, vol. 69, no. 5, pp. 696-729, 2001.

[22] G. Cohen, S. Gaubert, and J. Quadrat, "Duality and separation theorem in idempotent semimodules," Linear Algebra and Appl., vol. 379, pp. 395-422, 2004.

[23] V. N. Kolokoltsov and V. P. Maslov, "The general form of the endomorphisms in the space of continuous functions with values in a numerical commutative semiring (with the operation $\oplus=\max$ )," Dokl. Akad. Nauk SSSR, vol. 295, no. 2, pp. 283-287, 1987, engl. transl. in Sov. Math. Dokl., 36 (1), 55-59 (1988).

[24] V. Kolokoltsov, On linear, additive, and homogeneous operators, 1992, appeared in [5, p. 87-102].

[25] G. L. O'Brien and W. Vervaat, "Capacities, large deviations and $\log \log$ laws," in Stable processes and related topics, ser. Progress in probability, S. Cambanis, G. Samorodnitsky, and M. Taqqu, Eds., vol. 25. Birkhaüser, 1991, pp. 43-83.

[26] — - "Compactness in the theory of large deviations," Stochastic processes and their applications, vol. 57, pp. 1-10, 1995.

[27] T. Jiang and G. O'Brien, "The metric of large deviation convergence," J. Theoret. Probab., vol. 13, no. 3, pp. 805-824, 2000.

[28] C. C. W.M. McEneaney, "Large deviations theory, induced log-plus and max-plus measures and their applications," in Proceedings of MTNS'00, Perpignan, France, 2000.

[29] M. Akian, J.-P. Quadrat, and M. Viot, "Duality between probability and optimization," in Idempotency, J. Gunawardena, Ed. Cambridge University Press, 1998.

[30] S. R. S. Varadhan, Large Deviations and Applications, ser. CBMS-NSF Regional Conference Series in Applied Mathematics. Philadelphia, Penn.: SIAM, 1984, vol. 46.

[31] A. Puhalskiǔ, Large Deviations and Idempotent Probability, ser. Monographs and Surveys in Pure and Applied Mathematics. Chapman \& Hall, 2001, no. 119.

[32] W. Bryc, "Large deviations by the asymptotic value method," in Diffusion processes and related problems in analysis, M. A. Pinsky, Ed., vol. 1. Boston: Birkhaüser, 1990.

[33] R. T. Rockafellar, Convex analysis. N.J.: Princeton University Press Princeton, 1970.

[34] A. Puhalskii, "The method of stochastic exponentials for large deviations," Stochastic processes and their applications, vol. 54, pp. 45-70, 1994.

[35] O. V. Gulinsky, "The principle of the largest terms and quantum large deviations," Kybernetika, vol. 39, no. 2, pp. 229-247, 2003.

[36] R. C. Merton, "Optimum consumption and portfolio rules in a continuous time model," J. Economic Theory, vol. 3, pp. 373-413, 1971. 